Progress in Flight Physics 5 (2013) 481-492

DOI: 10.1051 /eucass/201305481

(C) Owned by the authors, published by EDP Sciences, 2013

\title{
NUMERICAL SIMULATIONS OF SUPERSONIC FLOW PAST THE EARTH DESCENT MODULE FOR PHOBOS-GRUNT SAMPLE RETURN MISSION
}

\author{
I. V. Egorov, D. V. Ivanov, and A. V. Novikov \\ Central Aerohydrodynamic Institute (TsAGI) \\ Zhukovsky, Moscow Region 140180, Russia
}

The results of numerical modeling of a steady viscous compressible flow around The Earth descent module for Phobos-grunt interplanetary mission at Mach numbers 10 and 3 are presented.

\section{INTRODUCTION}

The Russian sample return mission "Phobos grunt" is planned to study the Mars's moon Phobos and deliver a soil sample to the Earth. The mission was scheduled to launch late 2011. The Earth descent module will detach from the return vehicle and will enter the Earth atmosphere with the second cosmic speed. The descent module is an axis-symmetric blunt body without any flight controls.

The design of the descent vehicle in the Scientific Development and Production Center named after Lavochkin was accompanied by theoretical and experimental studies. The construction, shape, and the systems of the descent vehicle were coordinated with the changes of the overall arrangement of the spacecraft Phobos-grunt, caused by the design of all components of systems, modules, units, and elements.

Theoretical engineering calculations provided the determination of design parameters of the descent vehicle at preliminary stages of design. Following the results of experimental investigations and more detailed calculations using full gas motion equations, it was decided either to accept or to correct each of the engineering decisions made as a result of the theoretical research or laboratory and model tests.

After choosing the shape, the estimation of design parameters of the descent vehicle aerodynamics at some characteristic points of the descent trajectory in

This is an Open Access article distributed under the terms of the Creative Commons Attribution License 2.0, which permits unrestricted use, distribution, and reproduction in any medium, provided the original work is properly cited. 
the Earth atmosphere is fulfilled in the present paper. On the estimated descent trajectory, the module will pass regimes when the free stream is supersonic flow of a continuous medium (air).

In the present work, the results of numerical modeling of a steady viscous compressible flow around the module at Mach numbers 10 and 3 are presented. The three-dimensional (3D) Navier-Stokes and Reynolds equations are solved numerically using the in-house code HSFlow which implements the conservative second-order total variation diminishing (TVD) shock-capturing scheme coupled with the second-order fully implicit temporal advance method. Computations are carried out on a single-block curved orthogonal grid with $101 \times 51$ $\times 41$ nodes.

The calculations show that solutions of Navier-Stokes and Reynolds equations lead to similar aerodynamic characteristics, i.e., the laminar-turbulent transition do not address the flow cases considered. It is also shown that coefficients of lift and drag forces obtained from the flow fields calculated in this work using full Navier-Stokes equations are in a good agreement with estimations via a simplified engineering method by the descent module developers.

\section{PROBLEM FORMULATION}

\subsection{Differential Equations}

To simulate a continuous medium flow over a descent module, the computer software package HSFlow worked out at TsAGI is used. The flow simulation method is based on the integration of Navier-Stokes and Reynolds equations.

At zero angle of attack, a numerical simulation was carried out based on the unsteady two-dimensional (2D) axisymmetric Navier-Stokes equations under the assumption of axial symmetry of the flow field and free-stream homogeneity.

At nonzero angle of attack, a simulation on the basis of unsteady 3D NavierStokes equations was carried out under the assumption of the flow field symmetry relative to the vertical plane and free stream homogeneity. In this case, the computational area represents a half of the full $3 \mathrm{D}$ field.

The system of differential equations is closed by the state equation and dependencies of transfer coefficients on temperature and pressure whose form depends on the moving medium model. In the case of the perfect gas model with a state equation

$$
p=\frac{\rho R T}{M}
$$


where $R$ is the universal gas constant and $M$ is the gas molar weight, the molecular viscosity coefficient depends only on the temperature and is calculated according to the power law $\left(\mu / \mu_{0}=\left(T / T_{\infty}\right)^{\omega}, \omega=0.7\right)$, with the Prandtl number $\operatorname{Pr}=\mu c_{p} / \lambda=0.7$ taken to be constant.

The Navier-Stokes equations are solved in dimensionless quantities: Cartesian coordinates $x=\bar{x} L, y=\bar{y} L$, and $z=\bar{z} L$ are related to the characteristic linear size $L$; the time $t=\bar{t} L / V_{\infty}$ is related to the characteristic time $L / V_{\infty}$; the velocity vector components, $v=\bar{v} V_{\infty}$, and $w=\bar{w} V_{\infty}$, are related to the absolute value of the free-stream velocity vector $V_{\infty}$; the pressure $p=\bar{p}\left(\rho_{\infty} V_{\infty}^{2}\right)$ is related to the twice the impact air pressure of the free stream; and other gasdynamic variables are related to their values in the free stream. The upper stroke over a symbol means that this variable is dimensionless, and the symbol $\infty$ means the value of this variable in the free stream.

At these dimensionless variables, the main similarity parameters appear in the Navier-Stokes equations: $\gamma=c_{p} / c_{v}$ is the specific heat ratio; $\mathrm{M}_{\infty}=V_{\infty} / a_{\infty}$ is the free-stream Mach number ( $a$ is the sound velocity); $\operatorname{Re}_{\infty}=\rho_{\infty} V_{\infty} L / \mu_{\infty}$ is the Reynolds number; and Pr is the Prandtl number.

\subsection{Boundary and Initial Conditions}

Imposed on the computational area boundary, coinciding with a solid surface, are no-slip and no-leakage conditions $u=v=w=0$ and the condition of balance between the body radiation and heat flux to the surface (at hypersonic overflow):

$$
\left[\lambda \frac{\partial T}{\partial n}\right]_{w}=k \sigma\left(T_{w}^{4}-T_{\infty}^{4}\right)
$$

where $k=0.9$ is the body emissivity factor, and $\sigma=5.67040 \cdot 10^{-8} \mathrm{~J} \cdot \mathrm{s}^{-1} \mathrm{~m}^{-2} \mathrm{~K}^{-4}$ is the Stefan-Boltzmann constant.

Imposed on the outer (with respect to the body surface) boundary are the radiation conditions, corresponding to the diverging wave.

Used as an initial approximation is a field, calculated in the axisymmetric statement, with subsequent development of flow field while solving unsteady problem. In this case, as the flow pattern forms, the time step gradually increases that ultimately makes it possible to solve the steady problem.

\subsection{Numerical Method}

The systems of Navier-Stokes and Reynolds equations are integrated numerically using implicit finite volume method with a second order approximation in 
space and time. A quasi-monotone Godunov-type scheme (TVD scheme) with a limiter minmod is applied. This gives at each time step a system of nonlinear algebraic equations, which is solved by means of the iterative Newton technique.

At each iteration, the corresponding linear system is solved using the Generalized Minimal Residual (GMRes) method. This approach will be the most effective, if the computational area contains the shock waves and other strong spatial inhomogeneities, such as boundary layer separations.

The numerical method pointed out was verified and widely used while solving a number of supersonic problems of external and internal aerodynamics (circular cylinder, sphere, cone, flat and axisymmetric channels) both in 2D [1-3] and in $3 \mathrm{D}[4,5]$ statements.

\subsection{Computational Grid}

Calculations are fulfilled on curvilinear orthogonal grids, containing $101 \times 101$ and $201 \times 201$ nodes in the $2 \mathrm{D}$ case and $101 \times 51 \times 41$ nodes in the $3 \mathrm{D}$ case.

The $2 \mathrm{D}$ grid is constructed using numerical conformal mapping of the rectangle to the computational area, that gives a high degree of orthogonality (Fig. 1). It is refined near the surface to resolve the boundary layer.

The grid in the 3D computational area is obtained by rotating the flat grid around the vehicle axis (Fig. 2).

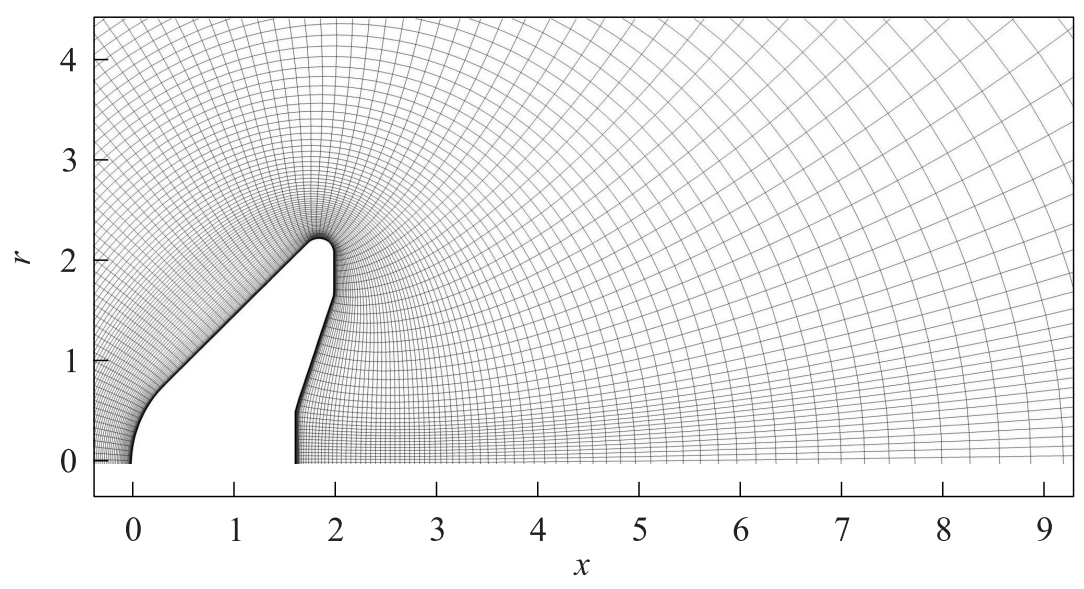

Figure 1 Fragment of the computational grid in the symmetry plane 

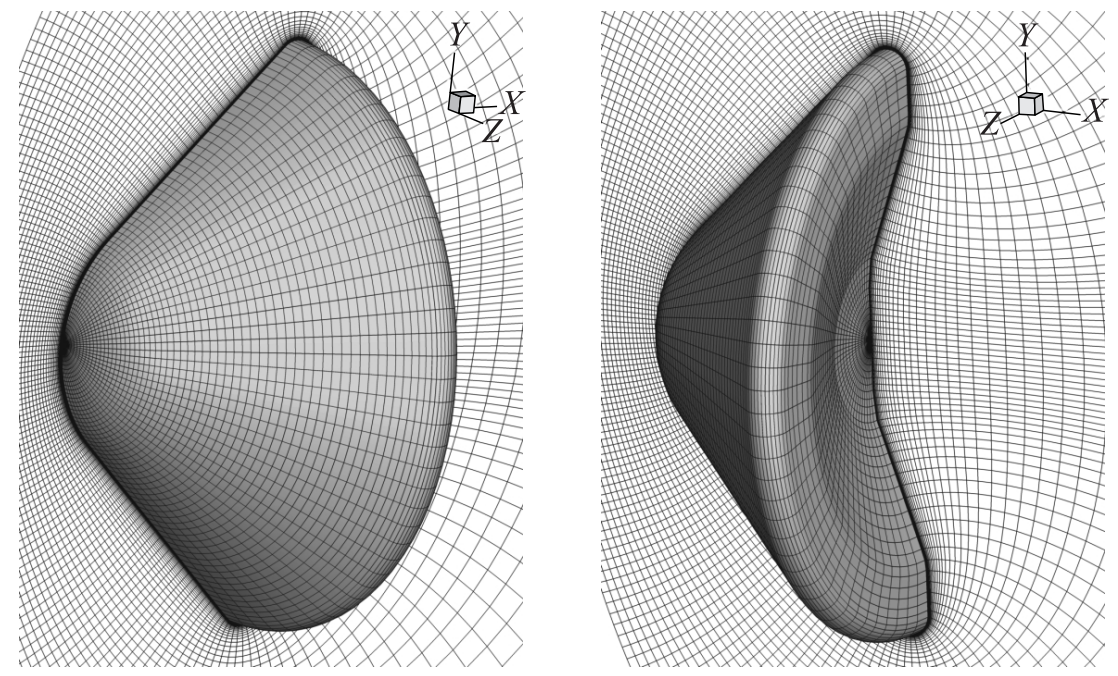

Figure 2 Fragments of the 3D computational grid around the descent module

\section{AERODYNAMIC CHARACTERISTICS}

The flow regime with the free-stream Mach number $\mathrm{M}_{\infty}=10.0$ corresponds to the point of the descent module trajectory at a height $H=32.5 \mathrm{~km}$. The following free-stream parameters corresponding to the nominal atmosphere at this height were used in the calculations: $T_{\infty}=242.25 \mathrm{~K}, \rho_{\infty}=0.0130 \mathrm{~kg} / \mathrm{m}^{3}, \mu_{\infty}$ $=0.0012506 \mathrm{~m}^{2} \mathrm{~s}, a=312.02 \mathrm{~m} / \mathrm{s}$, and unit-Reynolds number $\mathrm{Re}_{1}=0.33$. $10^{6} \mathrm{~m}^{-1}$. The radiation balance condition by the Stefan-Boltzmann law is imposed on the surface. For each case, several different angles of attack $\left(0^{\circ}, 3^{\circ}, 6^{\circ}\right.$, $9^{\circ}, 12^{\circ}, 15^{\circ}$, and $18^{\circ}$ ) are investigated.

For the regime of the flow over the descent module at zero angle of attack, the test calculations based on the $2 \mathrm{D}$ axisymmetric equations both with account of turbulence (Reynolds equations) and without it (Navier-Stokes equations) are performed. Mach number field is shown in Fig. 3. The analysis of the calculated data shows that the flow fields differ slightly, as expected, because for the given Reynolds number, the flow over the descent module should be mostly laminar. The analysis of the distribution of the pressure coefficient $C_{p}$ along the vehicle generatrix also shows that the calculation results based on the Navier-Stokes and Reynolds equations differ insignificantly.

Therefore, a simulation of flow over the descent module at this regime can be carried out based on the numerical solution of Navier-Stokes equations, which are less demanding of computational resources, as compared to the Reynolds equations with two-parameter differential turbulence model. 
M
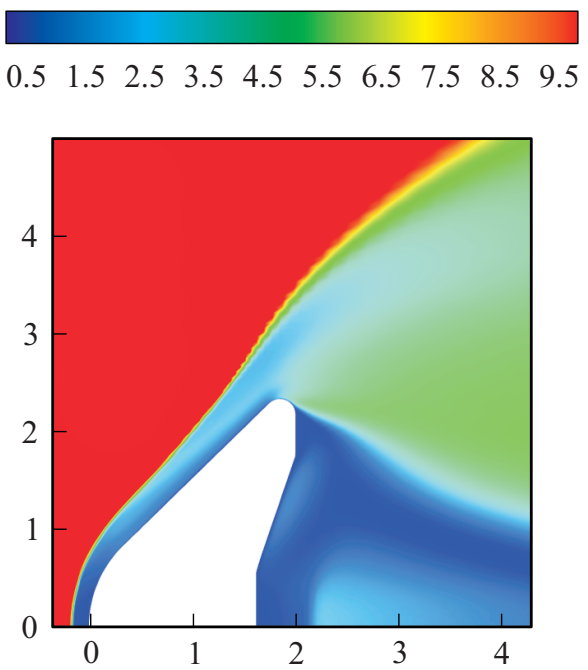

Figure 3 Mach number field at axisymmetric flow over the descent module, $\mathrm{M}_{\infty}$ $=10.0$.

The numerical simulation based on the Navier-Stokes equations in 3D statement is performed for the regime of the flow over the descent module at zero angle of attack. For the considered calculation conditions, a laminar flow of perfect gas with a complex structure of flow field characterized by the presence of shock waves, rarefaction waves and closed separation areas is implemented near the descent module. Particularly, the overall structure of the flow field can be estimated by the patterns of Mach number field in the symmetry plane and of the pressure distribution along the descent module surface for various angles of attack as presented in Fig. 4.

Since for strongly blunt bodies friction forces make a small contribution to the drag, for the qualitative analysis of aerodynamic characteristics, only the pressure behavior on the streamlined surface of the descent module can be considered. Patterns of the dimensionless pressure fields give a general idea of the pressure coefficient distribution along the body streamlined surface (Fig. 5). The presence of an angle of attack leads to the disarrangement of the axial symmetry in the pressure coefficient distribution on the body front surface.

The regime of the flow with the free stream Mach number $\mathrm{M}_{\infty}=3.0$ corresponds to the point of the descent module trajectory at a height $H=28.0 \mathrm{~km}$. Used in the calculations are the free stream parameters, corresponding to the nominal atmosphere at this height: $T_{\infty}=230.53 \mathrm{~K}, \rho_{\infty}=0.0260 \mathrm{~kg} / \mathrm{m}^{3}, \mu_{\infty}$ $=0.00057572 \mathrm{~m}^{2} \mathrm{~s}, a=304.38 \mathrm{~m} / \mathrm{s}$, unit-Reynolds number $\operatorname{Re}_{1}-1.20 \cdot 10^{6} \mathrm{~m}^{-1}$. 

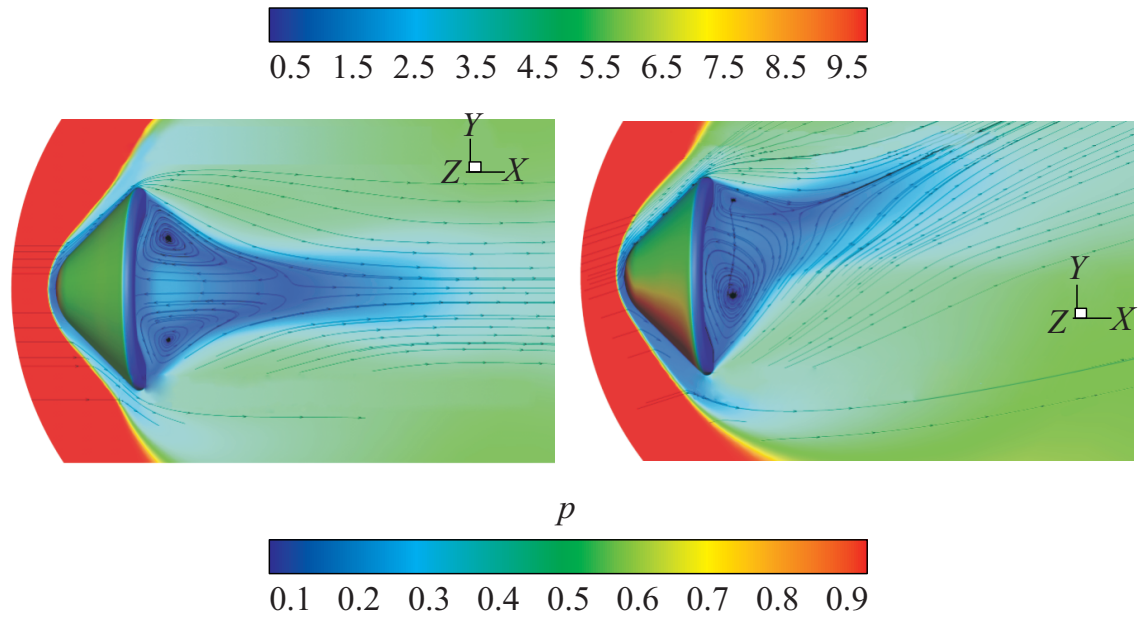

(a)

(b)

Figure 4 Mach number fields and streamlines in the flow symmetry plane, dimensionless pressure distribution along the descent module surface at $\mathrm{M}_{\infty}=10.0$ and angles of attack $\alpha=0^{\circ}(a)$ and $18^{\circ}(b)$.

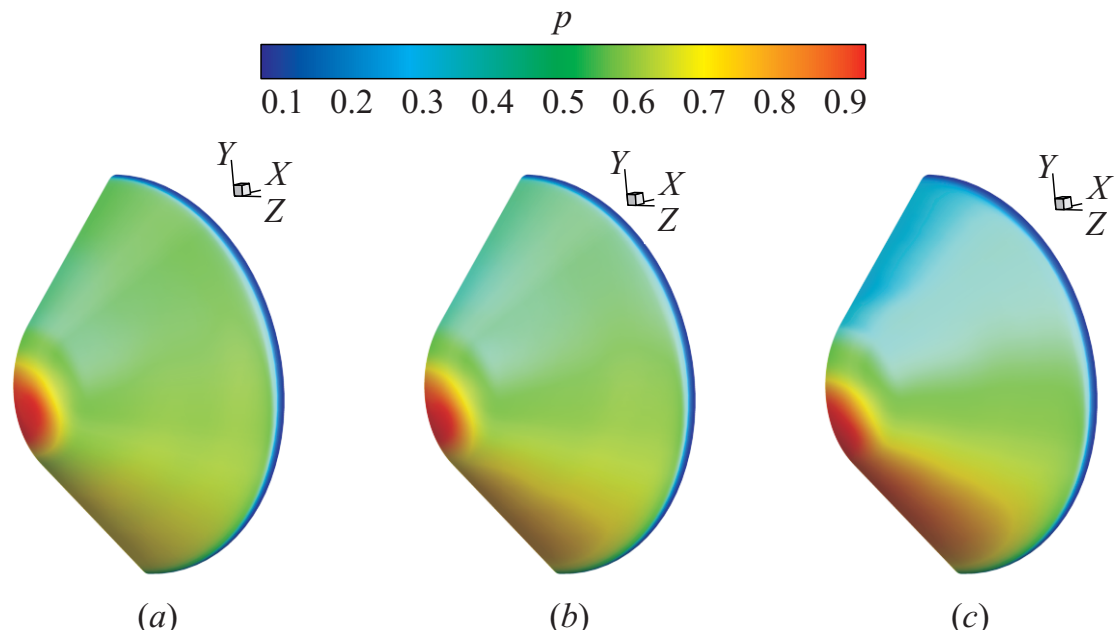

Figure 5 Dimensionless pressure field $\bar{p}=p /\left(\rho_{\infty} V_{\infty}^{2}\right)$ on the descent module surface at $\mathrm{M}_{\infty}=10.0$ and various angles of attack $\alpha:(a) 9^{\circ} ;(b) 12^{\circ}$; and $(c) 18^{\circ}$. 


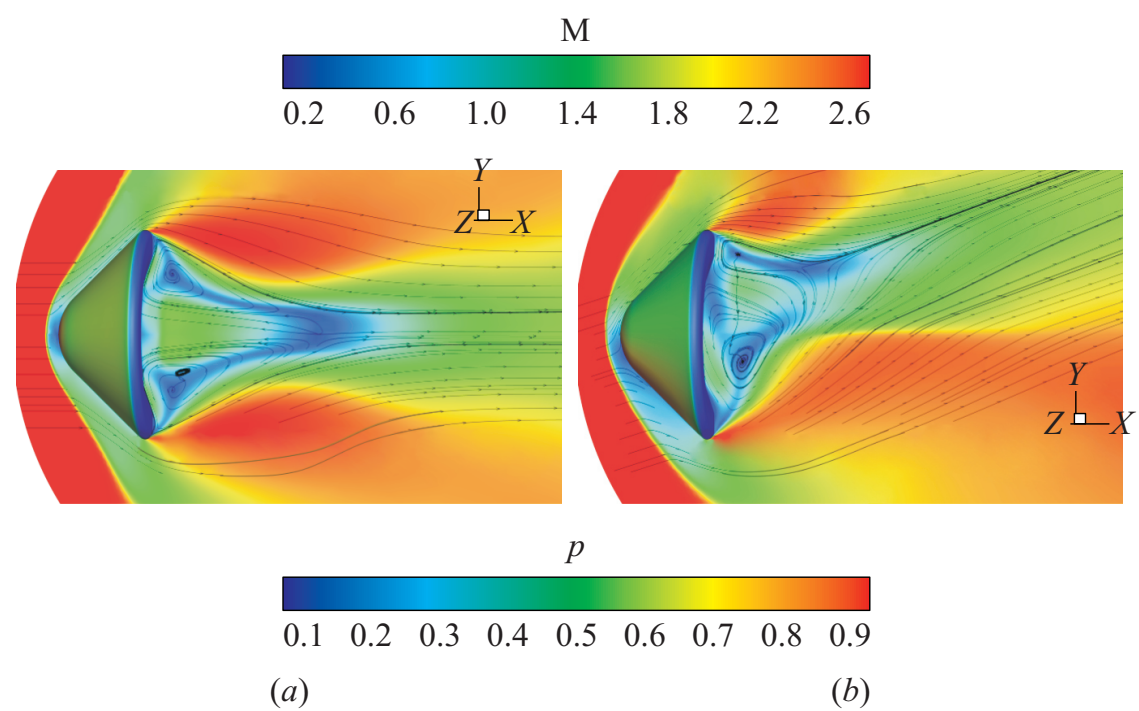

Figure 6 Mach number field and streamlines in the flow symmetry plane, dimensionless pressure distribution along the descent module surface at $\mathrm{M}_{\infty}=3.0$ and angles of attack $\alpha=0^{\circ}(a)$ and $18^{\circ}(b)$.

The radiation balance condition by the Stefan-Boltzmann law is imposed on the surface.

Calculations of the flow over the descent module are carried out for various angles of attack based on the solution of Navier-Stokes equations in the 3D statement. The overall structure of the flow field for two extreme angles of attack $\left(\alpha=0^{\circ}\right.$ and $\left.18^{\circ}\right)$ is shown in Fig. 6 in the form of Mach number field in the symmetry plane and of the pressure distribution along the descent module surface. Patterns of the dimensionless pressure fields give an idea of the pressure coefficient distribution along the body streamlined surface (Fig. 7).

Integral aerodynamic characteristics for the considered regimes of the flow over the descent module are presented in Fig. 8. Here, $\alpha$ is the angle of attack; $C_{A}$ is the coefficient of the axial component of the aerodynamic force; $C_{N}$ is the coefficient of the normal component of the aerodynamic force; and $C_{m}$ is the coefficient of the pitch moment relative to the center of mass $(x=0.131, y=0$, and $z=0)$. The midsection area $S=\pi D^{2} / 4$ and the shoulder $L=D$ where $D=0.450 \mathrm{~m}$ were used in normalization.

The results of calculating the variation of the descent module aerodynamic characteristics in the angle of attack obtained by means of the modified Newton method and by means of the solution of the Navier-Stokes equations are in a good agreement: for $\mathrm{M}=10$ and angle of attack equal to $15^{\circ}$, the aerodynamic 


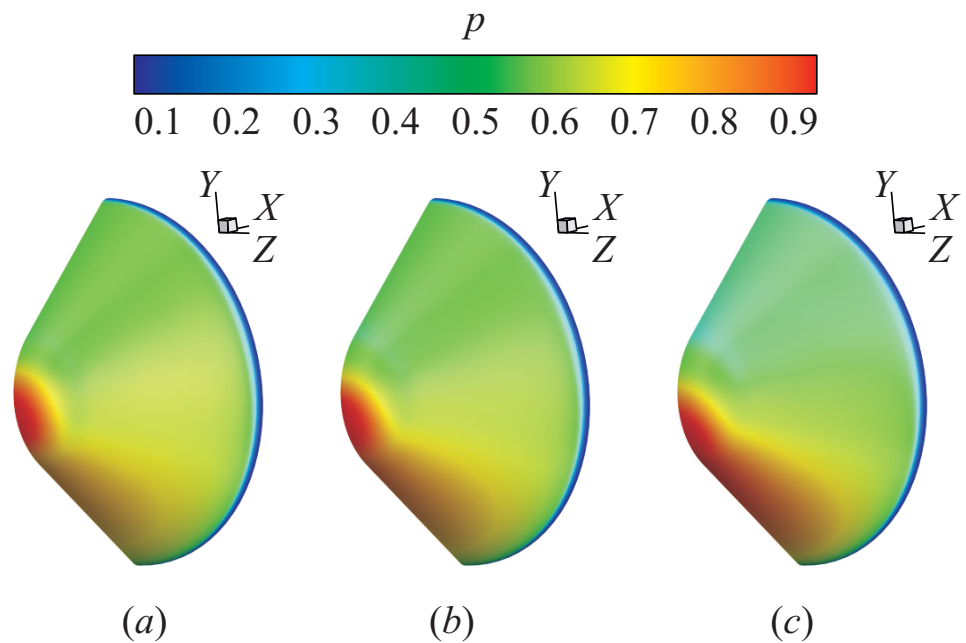

Figure 7 Dimensionless pressure field $\bar{p}=p /\left(\rho_{\infty} V_{\infty}^{2}\right)$ on the descent module surface at $\mathrm{M}_{\infty}=3.0$ and various angles of attack $\alpha:(a) 9^{\circ} ;(b) 12^{\circ}$; and $(c) 18^{\circ}$.

coefficients are $C_{A}=0.962, C_{N}=0.244$, and $C_{m}=-0.0695$, and by the Newton method, $C_{A}=1.000$ (relative difference is about $4 \%$ ), $C_{N}=0.246$ (about $1 \%$ ), and $C_{m}=-0.0712$ (about $2 \%$ ). In addition, the difference increases as the Mach number decreases, i. e., at the supersonic descent module, flight speed $(\mathrm{M}=3)$, the difference of the mentioned coefficients is more significant: $C_{A}=1.105$ (relative difference is about 7\%), $C_{N}=0.200$ (about 23\%), and $C_{m}=-0.0532$ (about 34\%).

The distribution of the pressure coefficient $C_{p}$ and the surface dimensionless temperature in the symmetry plane of the descent module for various angles of attack of the free stream is shown in Figs. 9 and 10, respectively. It is interesting that the pressure coefficient distribution is of a nonmonotonic nature on the front surface of the descent vehicle in the vicinity of the junction of the spherical and conical parts. The presence of a positive pressure gradient can lead to the boundary layer separation in the subsonic area of the flow on the windward part of the descent module. The separated flow can give rise to additional peaks in the heat flux in the vicinity of the mixing layer reattachment, as well as a premature laminar-turbulent transition. The latter phenomenon results also in a significant increase of the heat transfer.

The analysis of the calculated data presented in Fig. 9 shows a presence of quite a small pressure increase in the base part of the descent vehicle at small angles of attack caused by the stagnation of reverse streamlets in the separation 


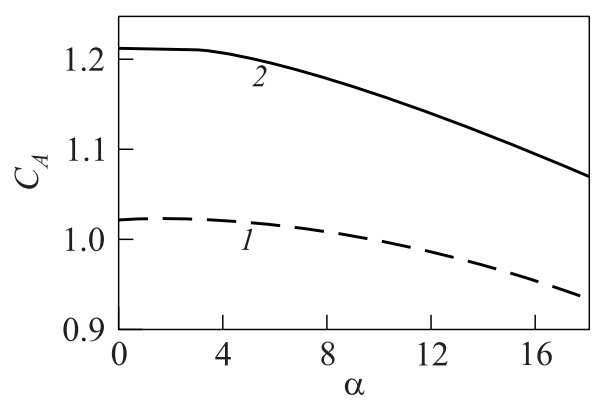

(a)

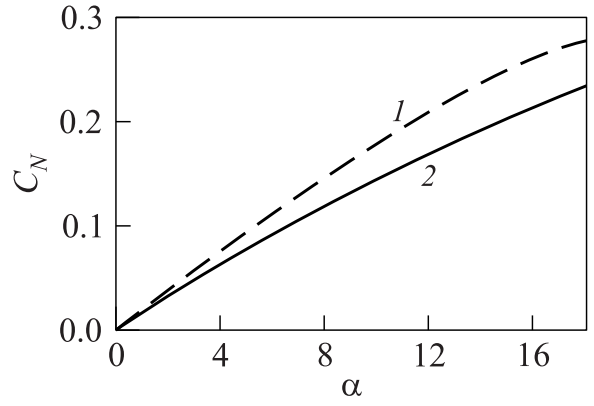

(b)

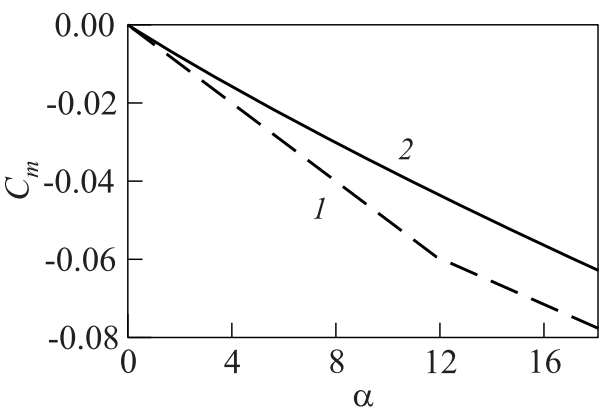

(c)

Figure 8 Coefficients of axial and normal forces, as well as of moment vs. free-stream angle of attack: $1-\mathrm{M}=10$; and $2-\mathrm{M}=3$

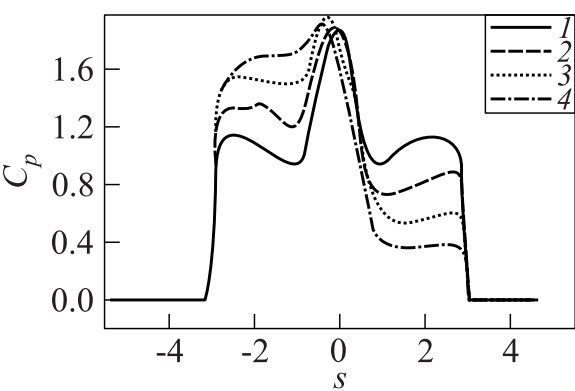

(a)

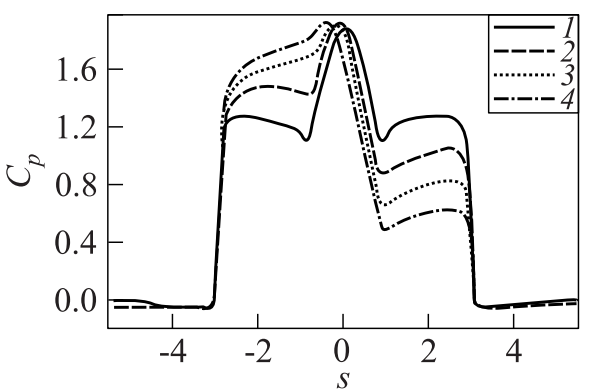

(b)

Figure 9 Distribution of pressure coefficient $C_{p}$ in the symmetry plane of the descent module for various free stream angles of attack $\left(1-\alpha=0^{\circ} ; 2-6^{\circ} ; 3-12^{\circ}\right.$; and $\left.4-\alpha=18^{\circ}\right):(a) \mathrm{M}=10$ and $(b) \mathrm{M}=3$ 


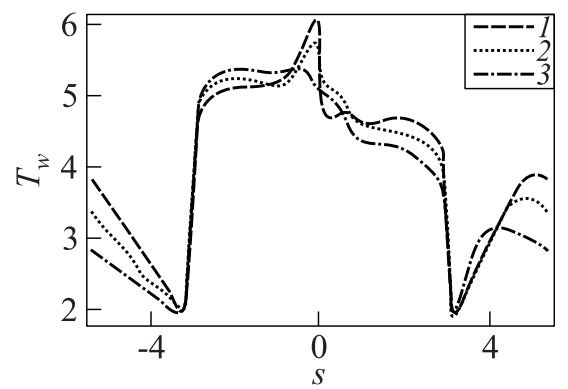

Figure 10 Distribution of dimensionless surface temperature $T_{w}$ in the symmetry plane of the descent module for various angles of attack $\left(1-\alpha=6^{\circ} ; 2-12^{\circ}\right.$; and $\left.3-\alpha=18^{\circ}\right) ; \mathrm{M}=10$

area. The shape of the descent vehicle surface having a concave form contributes to the pressure increase in the base area. This circumstance cannot influence significantly the behavior of forces and moments acting on the vehicle. However, it can lead to the heat transfer increase in its base area. The analysis of data on heating of the descent vehicle surface shown in Fig. 10 gives evidence of a significant increase of the surface temperature in its base part. In addition, the heat level depends substantially on the free stream angle of attack. At small angles of attack, it is much higher.

The analysis of calculated data of the present paper showed that in the area of supersonic flight speeds in the atmosphere, descent vehicle's aerodynamic characteristics should be specified on the basis of the numerical solution of NavierStokes and Reynolds equations or at wind tunnel tests of models geometrically similar to the descent module. At hypersonic part of the flight trajectory, the aerodynamic characteristics can be specified to an acceptable accuracy for engineering purposes by means of the Newton theory. However, while designing the descent vehicle, it is necessary to take into account aerodynamic heating of the base part of the given descent vehicle configuration at the hypersonic part of the flight trajectory.

\section{ACKNOWLEDGMENTS}

This work is supported by the Russian Foundation for Basic Research (grants Nos. 10-01-91332, 11-08-01099) and Federal goal-oriented program "Scientific and scientific-pedagogical personnel of innovative Russia" (state contracts Nos. 02.740.11.0154, 02.740.11.0203, and P595). 


\section{REFERENCES}

1. Bashkin, V.A., I. V. Egorov, and M. V. Egorova. 1993. Supersonic viscous perfect gas flow past a circular cylinder. Fluid Dyn. 28(6):833-38.

2. Bashkin, V. A., I. V. Egorov, and D. V. Ivanov. 1997. Application of the Newton method to the calculation of internal supersonic separated flows. J. Appl. Mech. Techn. Phys. 38(1):26-37.

3. Bashkin, V. A., I. V. Egorov, M. V. Egorova, and D. V. Ivanov. 2000. Supersonic laminar-turbulent gas flow past a circular cylinder. Fluid Dyn. 35(5):652-62.

4. Bashkin, V.A., I. V. Egorov, D. V. Ivanov, and V. V. Pafnutiev. 2002. Threedimensional laminar supersonic gas flow over the axis-symmetric bodies. Comput. Math. Math. Phys. 42(12):1864-74.

5. Bashkin, V. A., I. V. Egorov, D. V. Ivanov, and V. I. Plyashechnik. 2003. Theoretical and experimental investigation of supersonic flow past a slender sharp circular cone at incidence. Fluid Dyn. 1. 